\title{
Adherence to Antihypertensive Treatment and Associated Factors in Central Ethiopia
}

\author{
Daniel G/Tsadik (D), ${ }^{1}$ Yemane Berhane, ${ }^{2}$ and Alemayehu Worku ${ }^{3}$ \\ ${ }^{1}$ Department of Nursing, School of Health Sciences, Arsi University, Asella, Ethiopia \\ ${ }^{2}$ Addis Continental Institute of Public Health, Addis Ababa, Ethiopia \\ ${ }^{3}$ School of Public Health, Addis Ababa University, Addis Ababa, Ethiopia \\ Correspondence should be addressed to Daniel G/Tsadik; danielt@arsiun.edu.et
}

Received 25 April 2020; Revised 28 September 2020; Accepted 8 October 2020; Published 23 October 2020

Academic Editor: Tomohiro Katsuya

Copyright (c) 2020 Daniel G/Tsadik et al. This is an open access article distributed under the Creative Commons Attribution License, which permits unrestricted use, distribution, and reproduction in any medium, provided the original work is properly cited.

\begin{abstract}
Background. Adherence to treatment is a primary determinant of treatment success. Nonadherence attenuates clinical benefits to the recipients of the treatment. However, monitoring adherence to long treatment regimens is not given due emphasis in lowincome contexts. This study aimed to assess adherence to antihypertensive treatment and factors associated with it in Central Ethiopia. Method. This is an institution-based cross-sectional study conducted in 4 public hospitals in Central Ethiopia from December 4, 2016, to September 25, 2017. The Morisky Medication Adherence Scale (MMAS-8) was used to assess the level of adherence. The Revised Illness Perception Questionnaire (IPQ-R) was used to assess illness perception. The MMAS- 8 score ranges from 0 to 8 , a score of 8 reflects high adherence, 6 to 7 medium adherence, and $<6$ low adherence. Results. A total of 989 hypertensive patients participated in the study, of which $36.0 \%$ were assessed to have high adherence, $31.7 \%$ medium adherence, and $32.3 \%$ low adherence. We found that treatment adherence was significantly and positively associated with having family support (AOR: $1.65 ; 95 \% \mathrm{CI}=1.23,2.22$ ), high perception about consequences of hypertension (AOR: $1.51 ; 95 \% \mathrm{CI}=1.17,1.95$ ), and high perception about the severity of the disease (AOR: 1.42; 95\% CI $=1.09,1.86$ ). Conclusion. The treatment adherence to antihypertensive medications is low in Central Ethiopia. Engaging family members in the treatment plan and improving patients' understanding of the illness are critical in achieving high adherence to medication in this context.
\end{abstract}

\section{Introduction}

Hypertension (HTN) is one of the major public health problems globally $[1,2]$, in both high-income as well as lowand middle-income countries [3]. However, awareness about the disease and its prevention and treatment are low in lowand middle-income countries compared to developed countries [3]. According to the Global Burden of Disease Study, hypertensive heart disease accounts for 17.5 million disabilityadjusted life years in 2015 [4]. Optimal control of blood pressure is paramount to prevent hypertension-related complications and deaths [5]. High adherence to antihypertensive medications can effectively lower hypertension-related complications and improve survival $[6,7]$.
Medication adherence is defined as "the extent to which the medication-taking behavior of a patient corresponds with agreed recommendations/prescriptions." Patients receiving medication need to understand that the medications are critical to achieving blood pressure control $[8,9]$. Many studies have suggested that a high level of adherence to antihypertensive drug treatment is related to better blood pressure (BP) control and a reduced risk of cardiovascular disease (CVD) $[8,10-13]$; however, low adherence to antihypertensive drugs is a major public health and clinical challenges in the treatment of hypertension in low- and middle-income countries [14, 15]. Patients who poorly adhere to antihypertensive medications have a higher risk of adverse outcomes, including hospitalization, and incur 
higher healthcare costs due to complications as compared to patients who had good adherence [12, 13, 16, 17].

Multiple factors including patients' beliefs about health, illness, and treatment contribute to antihypertensive medication adherence therapy [18-23]. In treating hypertension, understanding patient's beliefs about medication adherence is fundamental because hypertension is silent and asymptomatic. Thus, patients might have misperceptions about hypertension, its severity, and the significance of its management $[24,25]$. Socioeconomic status (poverty), low level of education, unemployment, lack of effective family/social support, and forgetfulness are also associated with adherence [26]. The patients' illness representations also have a direct influence on adherence to treatment [27].

Although the importance of treatment adherence has been recognized in Ethiopia by prior studies, the previous studies were conducted on a small number of patients often drawn from a single hospital and gave emphasis mostly only to sociodemographic factors [28-32]. Important independent factors that could influence patients' adherence to their treatment such as illness perception and health belief were hardly studied in Ethiopia. Hence, this study assessed the magnitude of antihypertensive treatment adherence and factors associated with it in multiple hospitals in Central Ethiopia.

\section{Material and Methods}

2.1. Study Design and Setting. This is a hospital-based crosssectional study conducted from December 4, 2016, to September 25,2017 . The study was conducted in 4 public hospitals, namely, Bishoftu, Adama, Asella, and Shashemene. These hospitals are located in Oromia Regional State in Central Ethiopia. The hospitals provide a comprehensive healthcare service for both outpatient and inpatient clients. These hospitals also have specialty clinics where patients with specific chronic diseases are referred for follow-up. The hypertension clinic is one of those clinics. In Ethiopia, prescriptions are written by a physician in the follow-up clinics and patients get their medications from pharmacies from the same facility or pharmacies outside the healthcare facilities.

2.2. Study Population. All adults following their antihypertensive treatment in the four study hospitals were and who fulfilled the inclusion criteria were included in the study. The inclusion criteria include age at least 18 years or above, a diagnosis of hypertension confirmed by a physician, patients on antihypertensive medication for at least 3 months, patients who can give consent to participate in the study, and patients with no acute distress related to any disease during recruitment of study participants. Pregnant women, patients who cannot give consent, and patients who have hearing and/or speaking problems were excluded from the study.

2.3. Sample Size and Sampling Procedures. The sample size for determining the proportion of adherence to hypertensive treatment was determined with the following assumption: adherence to antihypertension treatment taken as $64.6 \%$ based on Ambaw et al. study [28], a 4\% precision, 95\% level of confidence, and $10 \%$ nonresponse rate. Accordingly, the calculated sample size was 604 . For factors associated with adherence to antihypertensive treatment, we used a research conducted by [28]. Using StatCalc, the most determinant factor for treatment adherence was residence of the patients and considering $P_{1}=28.7, \mathrm{OR}=0.64,80 \%$ power, $95 \%$ confidence level, and 1:1 $\left(n_{1}=n_{2}\right)$ ratio. After adding 10\% nonresponse, the calculated sample size was 1030 (515 urban and 515 rural). Hypertensive patients who consented for the study were consecutively enrolled until the required sample was fulfilled.

2.4. Data Collection. Data were collected using a uniform and pretested questionnaire by 12 trained nurses ( 3 for each hospital). The study nurses were not working at the hypertension clinic. Data were collected through a face-to-face interview. The data collectors were competent to do interviews either in Amharic (the national language) and Afaan Oromo (the state language). Additionally, one nurse was assigned to supervise the data collection process at each hospital. Patients were interviewed after they got their routine services at existing. Before collecting data, the data collectors explained the objective of the study and obtained informed verbal consent from each study participant. The data collection tool contained questions on sociodemographic information, lifestyle, health-related matters, illness perception (IPQ-R) [33], the Morisky Medication Adherence Scale (MMAS-8) [34], and health beliefs. Some of these components are described in more detail below.

2.5. Illness Perception Questionnaire-Revised (IPQ-R). The Illness Representation (IR) component of the validated IPQ$\mathrm{R}$ [33] had seven subscales and 34 questions including the subdimensions of the timeline, timeline cyclical or symptoms fluctuate over time, consequences, personal control, treatment control, illness coherence, and emotional representations. In all dimension's subjects were given 5 options which were converted to a 5-point Likert-type scale for result analysis: strongly disagree (1), disagree (2), neither agree nor disagree (3), agree (4), and strongly agree (5).

For all these subscales, partial scores were defined as the mean of the scores for the items on each subscale (considering direct and inverse items, eight negative item scores were reversed: $1,6,13,15,19,22,23$, and 32). Then, responses of all items are summed according to the IR dimensions ( 4 items of the timeline, 4 items of timeline cyclical, 6 items of consequences, 4 items of personal control, 5 items of treatment control, 5 items of illness coherence, and 6 items of emotional perceptions) and then dichotomized in to high and low by their mean. A higher score indicates stronger beliefs about the disease chronicity, cyclical course, impact and outcomes, personal influence, cure possibilities, perceived understanding, and emotional reactions to the disease. The internal consistency of Cronbach's alpha of the IPQ-R was checked and was 0.78, indicating satisfactory internal consistency. 
2.6. Hypertension Belief and Behavior Questionnaire. The questionnaire is generated by referring to previously published studies based on the constructs of the HBM [35]. The HBM consisted of perceived susceptibility, perceived severity, perceived benefits, perceived barriers, perceived selfefficacy, and cues to action. The scores of each one of the HBM constructs were evaluated based on a 5-item Likert scale. Perceived susceptibility, perceived severity, perceived benefits, perceived barriers, perceived self-efficacy, and cues to action consisted of $4,5,5,5,4$, and 4 questions, respectively.

2.7. Adherence to Medication. Medication adherence was measured using a validated eight-item self-reported Morisky Medication Adherence Scale (MMAS-8) [34]. Each item measures specific medication-taking behavior. Approval was obtained from professor Morisky to use his scale. The questions are phrased to avoid the "yes-saying" bias by reversing the wording of the questions about the way patients might experience failure in following their medication regimen since there is a tendency for patients to give their physicians or other healthcare providers positive answers [34].

This scale is a questionnaire with high reliability and validity, which has been particularly useful in chronic conditions such as hypertension. Response choices were "Yes" or "No" for items 1 through 7, and item 8 has a fourpoint Likert response scale. Total scores on the MMAS-8 ranged from 0 to 8 , with scores of 8 reflecting high adherence, 6 to $<8$ reflecting medium adherence, and $<6$ reflecting low adherence. The internal consistency of Cronbach's alpha of the MMAS was checked and was 0.72 $[34,36,37]$.

2.8. Statistical Analyses. Descriptive statistics were used to summarize sociodemographic, disease characteristics of the study population, and the nature and frequency of antihypertensive medications used. We examined sociodemographic, health-related variables, illness representation variables, and health belief items considering adherence as an outcome variable. The variables which have a $P$. value of 0.25 in bivariate analysis entered into multivariable ordinal logistic regression analysis. The ordinal logistic regression model was used because the outcome variable had three ordered levels of adherence (low/ medium/high). This model compared adjacent levels: the odds ratio (OR) corresponded with the odds of adherence to the next lower level. The ORs are presented with their $95 \%$ confidence intervals (CIs). The level of significance was set at a $P$ value of less than 0.05 . STATA 12 software was used for data entry and analyses.

\section{Result}

A total of 989 study participants were interviewed from four public hospitals in Central Ethiopia. The response rate was $96.0 \%$. The mean age of respondents was 57.6 years $(\mathrm{SD}=11.8)$. Orthodox Christians and the Oromo ethnic group accounted for $64.6 \%$ and $51 \%$ of the respondents, respectively. More than three-fourth (76.8\%) of the respondents were urban residents. Six hundred twenty-seven $(63.4 \%)$ reported to be married, $34.6 \%$ were illiterate, and $32.9 \%$ were housewives (Table 1 ).

About three-fourth (73.8\%) of the respondents reported that it takes half an hour or more for a single trip to reach the hospital where they are receiving antihypertensive treatment. More than half $(55.7 \%)$ of the respondents visit the hospital once every month; about three-fourth $(72.7 \%)$ did not have a family history of hypertension, and the majority (79.1\%) reported getting support from their family or friends (Table 2).

Concerning health-related conditions of the respondents, $42.8 \%$ were on treatment for about one to five years, $51.3 \%$ took two drugs daily, and about half (51.8\%) took their drugs more than once per day. Three-fourth $(75.1 \%)$ of the respondents reported no side effects of medications, $70.7 \%$ reported never heard about the side effects of the drug from healthcare providers, only $38.2 \%$ reported the cost of the drug is high, and the rest reported either fair or low. About half $(52.9 \%)$ of the respondents had comorbid chronic illnesses, and the most frequently reported comorbidity was diabetes mellitus by $80.1 \%(n=523)$. More than three-forth $(81.3 \%)$ of the respondents sleep for more than 6 hours with an average of 6.95 hours $(S D=1.98)$. The mean values of systolic blood pressure were $139.56(\mathrm{SD}=15.9) \mathrm{mmHg}$ and $86.3(\mathrm{SD}=8.32) \quad \mathrm{mmHg}$ for diastolic blood pressure (Table 3).

Concerning the respondent's illness representation, the mean scores of the seven dimensions of illness representation were $13.2 \pm 1.6$ for timeline, $13.2 \pm 2.2$ for coherence, $13.1 \pm 1.9$ for controllability-personal, $14.5 \pm 2.6$ for controllability-treatment, $20.4 \pm 2.8$ for a consequence, $11.1 \pm 3.1$ for timeline cyclical, and $17.9 \pm 4.3$ for emotional representation, respectively. This information indicates that patients believed that hypertension tended to be relatively chronic, predictable, and controllable through personal effort or by medical treatment and that hypertension had relatively little influence on their lives. Patients also believed that they had a relatively good understanding of their hypertension and rarely felt negative emotions due to hypertension (Table 4).

In this study, the researcher tried to assess hypertensive patient's beliefs using the health belief constructs. The findings indicated that respondents had lower beliefs of risk to contracting an illness or its complications (perceived susceptibility), lower beliefs that potential factors might make it difficult to take the behavior (perceived barriers), and lower internal or external cues that prompt the action, whereas they had higher beliefs that the disease is severe and has serious consequences (perceived severity), higher beliefs that taking treatment is beneficial to reduce or prevent disease threat (perceived benefit), and relatively good confidence to tackle their illness (self-efficacy) (Table 5).

Considering the frequency distribution of the MMAS- 8 items, the majority or more than three-fourth of the respondents reported positive responses for MMAS-8 items. However, MMAS-8 items sometimes forget taking their drugs and having some degree of remembering problem to 
TABLE 1: Sociodemographic characteristics of hypertensive patients in Central Ethiopia, $2017(n=989)$.

\begin{tabular}{|c|c|c|}
\hline Variable & Frequency & $\%$ \\
\hline \multicolumn{3}{|l|}{ Sex } \\
\hline Male & 468 & 47.3 \\
\hline Female & 521 & 52.7 \\
\hline \multicolumn{3}{|l|}{ Name of the hospital } \\
\hline Asella & 322 & 32.5 \\
\hline Adama & 268 & 27.1 \\
\hline Bishoftu & 246 & 24.9 \\
\hline Shashemen & 153 & 15.5 \\
\hline \multicolumn{3}{|l|}{ Age } \\
\hline $20-35$ & 43 & 4.3 \\
\hline $35-49$ & 241 & 24.4 \\
\hline $50-64$ & 512 & 51.8 \\
\hline $65-79$ & 193 & 19.5 \\
\hline \multicolumn{3}{|l|}{ Mean and SD $(57.6 \pm 11.8)$} \\
\hline \multicolumn{3}{|l|}{ Religion } \\
\hline Orthodox & 639 & 64.6 \\
\hline Muslim & 221 & 22.4 \\
\hline Protestant & 129 & 13.0 \\
\hline \multicolumn{3}{|l|}{ Address } \\
\hline Urban & 760 & 76.8 \\
\hline Rural & 229 & 23.2 \\
\hline \multicolumn{3}{|l|}{ Level of education } \\
\hline Illiterate & 342 & 34.6 \\
\hline Read and write & 148 & 14.9 \\
\hline Primary & 177 & 17.9 \\
\hline Secondary & 172 & 17.4 \\
\hline Diploma and above & 150 & 15.2 \\
\hline \multicolumn{3}{|l|}{ Marital status } \\
\hline Single & 154 & 15.6 \\
\hline Married & 627 & 63.4 \\
\hline Divorced & 55 & 5.5 \\
\hline Widowed & 153 & 15.5 \\
\hline \multicolumn{3}{|l|}{ Ethnicity } \\
\hline Oromo & 504 & 50.9 \\
\hline Amhara & 377 & 38.1 \\
\hline Tigre & 26 & 2.6 \\
\hline Gurage & 52 & 5.3 \\
\hline Others & 30 & 3.1 \\
\hline \multicolumn{3}{|l|}{ Occupation } \\
\hline GOV employed & 154 & 15.6 \\
\hline Self-employed & 151 & 15.3 \\
\hline Farmer & 129 & 13.0 \\
\hline Housewife & 325 & 32.9 \\
\hline Retired & 230 & 23.2 \\
\hline
\end{tabular}

take their medications reported less than three-fourth of the respondents, $63.3 \%$ and $52.3 \%$, respectively (Table 6 ).

Use of the OMMAS is protected by US copyright and registered trademark laws. Permission for use is required. A license agreement is available from MMAR, LLC., Donald E. Morisky, 294 Lindura Court, Las Vegas, NV 89138-4632, dmorisky@gmail.com.

Overall, 32.3\% (95\% CI: 29.1, 35.0) of participants demonstrated low adherence, 31.7\% (95\% CI: 29.0, 34.7) medium adherence, and 36.0\% (95\% CI: 33.0, 38.9) high adherence. About two-third (67.7\%) (95\% CI: 64.7, 70.7) of participants were either moderate or high adherent for their antihypertensive medications removed (Figure 1).
TABLE 2: Health-seeking and illness-related factors of hypertensive patients in Central Ethiopia, $2017(n=989)$.

\begin{tabular}{lcc}
\hline Variable & Frequency & $\%$ \\
\hline Frequency of visit & & \\
$\quad$ Once or less per month & 633 & 64.0 \\
$\quad$ Once in 2 months or more & 356 & 36.0 \\
Distance of residence & & \\
$\quad \leq 30$ minutes & 259 & 26.19 \\
$\quad>30$ minutes to $<1$ hour & 379 & 38.32 \\
$\quad \geq 1$ hour & 351 & 35.49 \\
Family history of hypertension & & \\
$\quad$ Yes & 234 & 23.7 \\
$\quad$ No & 755 & 76.3 \\
Presence of family support & & \\
$\quad$ Yes & 782 & 79.1 \\
$\quad$ No & 207 & 20.9 \\
\hline
\end{tabular}

In bivariate regression analysis, sex, educational status, occupation, frequency of visit, family support, perception on a timeline, perception on a consequence, cyclical perception, perceived susceptibility, perceived benefit, perceived severity, and cues to action had a significant association with treatment adherence.

After controlling possible confounding effects of other covariates, six factors remained as significant independent predictors of treatment adherence in the adjusted ordinal logistic regression model. These are farmer (AOR: 0.51; 95\% $\mathrm{CI}=0.33,0.79$ ), family support (AOR: $1.6 ; 95 \% \mathrm{CI}=1.23$, 2.22), perceived consequences of hypertension (AOR: 1.51; $95 \% \mathrm{CI}=1.17,1.95)$, perceived cyclical nature of hypertension (AOR: 0.76; 95\% CI $=0.58,0.95$ ), perceived susceptibility (AOR: $0.61 ; 95 \% \mathrm{CI}=0.48,0.78$ ), and perceived severity of the disease (AOR: 1.42; 95\% CI $=1.09,1.86$ ) had significant association with treatment adherence (Table 7).

\section{Discussion}

This study found that only about one-third of the respondents had a high level of treatment adherence. Being a farmer, having family support, higher perceived consequences, perceived cyclical nature of hypertension, perceived susceptibility, and perceived severity of the disease had a significant association with treatment adherence.

We collected the data for this study using an intervieweradministered questionnaire, which is a preferred method when conducting a fairly large survey among the community with substantial low literacy levels, to minimize cognitive burden and increase response rate [38]. To minimize social desirability bias to self-reported adherence, we used a standard questionnaire and carefully selected and trained data collectors to conduct the interviews in a nonjudgmental manner. Thus, the adherence level reported in this study is a fair reflection of the practice in the study areas.

In our study, higher adherence to hypertensive medication as measured by MMAS- 8 was only $36.0 \%$, which is comparable to several reports from low- and middle-income countries [39-43]. However, it is lower than some other studies in Ethiopia such as that reported from Gondar hospital, 64.6\% [28], Jimma Hospital, 55.7\% [30], in selected 
TABLE 3: Health-related conditions of hypertensive patients in Central Ethiopia, $2017(n=989)$.

\begin{tabular}{|c|c|c|}
\hline Variable & Frequency & $\%$ \\
\hline \multicolumn{3}{|c|}{ Duration on antihypertensive treatment } \\
\hline$<5$ years & 538 & 54.4 \\
\hline$\geq 5$ years & 451 & 45.6 \\
\hline \multicolumn{3}{|c|}{ Number of antihypertensive drugs taken } \\
\hline One & 429 & 43.4 \\
\hline More than one & 560 & 56.6 \\
\hline \multicolumn{3}{|c|}{ Antihypertensive drugs dosage/frequency } \\
\hline Once per day & 477 & 48.2 \\
\hline More than once & 512 & 51.8 \\
\hline \multicolumn{3}{|c|}{ Any history of side effect to antihypertensive drugs } \\
\hline Yes & 246 & 24.9 \\
\hline No & 743 & 75.1 \\
\hline \multicolumn{3}{|c|}{ Ever told about the side effect of antihypertensive drugs } \\
\hline Yes & 290 & 29.3 \\
\hline No & 699 & 70.7 \\
\hline \multicolumn{3}{|c|}{ Perceived cost of the antihypertensive drugs } \\
\hline Low & 113 & 11.4 \\
\hline Fair & 498 & 50.4 \\
\hline High & 378 & 38.2 \\
\hline \multicolumn{3}{|c|}{ Anything that makes you stressed } \\
\hline Yes & 523 & 52.9 \\
\hline No & 466 & 47.1 \\
\hline \multicolumn{3}{|c|}{ Duration of sleep in an hour } \\
\hline$<4$ hours & 55 & 5.5 \\
\hline 4 to 8 hours & 787 & 79.6 \\
\hline$>8$ hours & 147 & 14.9 \\
\hline \multicolumn{3}{|l|}{ Level of BP } \\
\hline Controlled & 415 & 41.9 \\
\hline Uncontrolled & 574 & 58.1 \\
\hline
\end{tabular}

TABLE 4: Illness perception of hypertensive patients in Central Ethiopia, $2017(n=989)$.

\begin{tabular}{|c|c|c|}
\hline Variable & Frequency & $\%$ \\
\hline \multicolumn{3}{|c|}{ Timeline acute/chronic } \\
\hline Low & 469 & 47.4 \\
\hline High & 520 & 52.6 \\
\hline \multicolumn{3}{|c|}{ Consequence } \\
\hline Low & 460 & 45.5 \\
\hline High & 529 & 53.5 \\
\hline \multicolumn{3}{|c|}{ Treatment control } \\
\hline Low & 428 & 43.28 \\
\hline High & 561 & 56.72 \\
\hline \multicolumn{3}{|c|}{ Personal control } \\
\hline Low & 422 & 43.3 \\
\hline High & 567 & 56.7 \\
\hline \multicolumn{3}{|c|}{ Illness coherence } \\
\hline Low & 544 & 55.1 \\
\hline High & 445 & 44.9 \\
\hline \multicolumn{3}{|c|}{ Timeline cyclical } \\
\hline Low & 495 & 50.1 \\
\hline High & 494 & 49.9 \\
\hline \multicolumn{3}{|c|}{ Emotional representation } \\
\hline Low & 523 & 52.9 \\
\hline High & 466 & 47.1 \\
\hline \multicolumn{3}{|c|}{ Illness representation } \\
\hline Low & 483 & 48.8 \\
\hline High & 506 & 51.2 \\
\hline
\end{tabular}


TABle 5: Hypertension belief and behavior of patients in Central Ethiopia, $2017(n=989)$.

\begin{tabular}{lcc}
\hline Variable & Frequency & $\%$ \\
\hline Perceived susceptibility & & \\
$\quad$ Lower & 588 & 59.5 \\
$\quad$ Higher & 401 & 40.5 \\
Perceived severity & 339 & \\
$\quad$ Lower & 650 & 34.3 \\
$\quad$ Higher & & 65.7 \\
Perceived benefits & 226 & \\
$\quad$ Lower & 763 & 77.2 \\
$\quad$ Higher & & \\
Perceived barriers & 518 & 52.4 \\
$\quad$ Lower & 471 & 47.6 \\
$\quad$ Higher & & \\
Cues to action & 563 & 56.9 \\
$\quad$ Lower & 426 & 43.1 \\
$\quad$ Higher & & \\
Self-efficacy & 478 & 51.7 \\
$\quad$ Lower & 511 & \\
$\quad$ Higher & &
\end{tabular}

TABLE 6: Responses of hypertensive patients to MMAS-8 questions in Central Ethiopia, $2017(n=989)$.

\begin{tabular}{lcc}
\hline Variable & Frequency & $\%$ \\
\hline Sometimes forget taking their drugs & & \\
$\quad$ Yes & 363 & 36.7 \\
$\quad$ No & 626 & 63.3 \\
Had a problem taking in the last 2 weeks & & \\
$\quad$ Yes & 164 & 16.6 \\
$\quad$ No & 825 & 83.4 \\
Stop taking or decrease the dose & & \\
$\quad$ Yes & 130 & 13.1 \\
$\quad$ No & 859 & 86.9 \\
Forget to bring along when traveling & & \\
$\quad$ Yes & 210 & 21.2 \\
$\quad$ No & 779 & 78.8 \\
Took their medication yesterday & & \\
$\quad$ Yes & 807 & 81.6 \\
$\quad$ No & 182 & 18.4 \\
Stop taking when they feel controlled & & \\
$\quad$ Yes & 149 & 15.1 \\
$\quad$ No & 840 & 84.9 \\
Felt hassled sticking treatment plan & & \\
$\quad$ Yes & 156 & 15.8 \\
$\quad$ No & 833 & 84.1 \\
Some degree of remembering a problem & & \\
$\quad$ Almost never & 517 & 52.3 \\
Sometimes & 408 & 41.2 \\
Frequently & 56 & 5.7 \\
Always & 8 & 0.8 \\
\hline
\end{tabular}

hospitals of Addis Ababa, 66.8\% [32], and Tikur Anbessa hospital in Addis Ababa, 69.2\% [31]. The possible reasons for this difference could be variations in the population groups and cutoff points to adherence between the studies. Another explanation might be due to differences in the study population characteristics, differences in sample size, and the area covered by the research. The present study covered

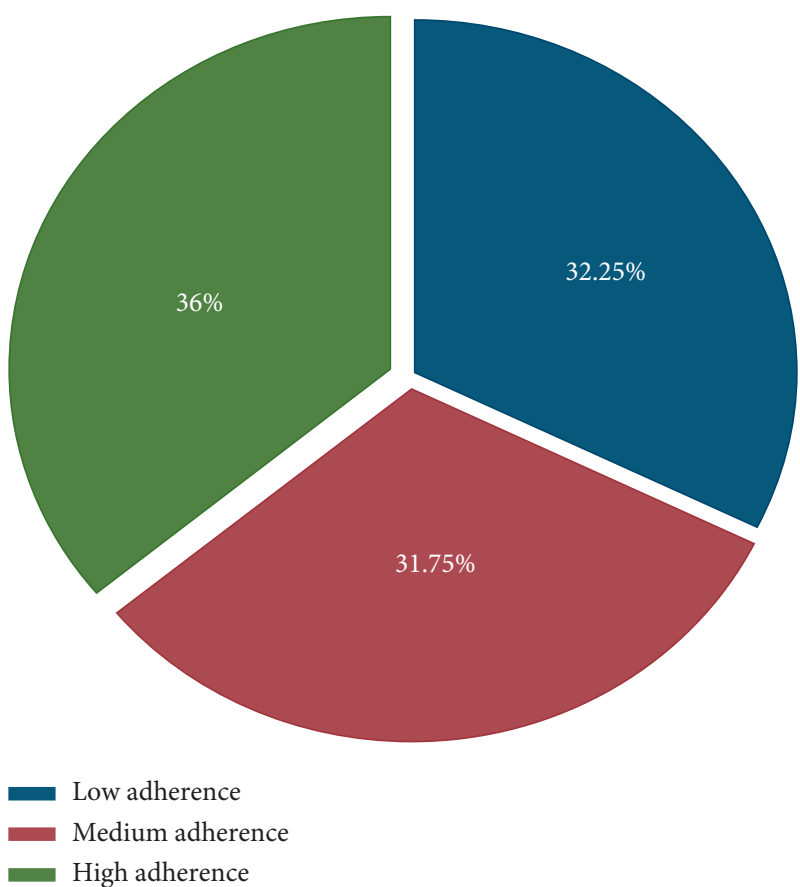

FIGURE 1: Hypertensive patient's level of treatment adherence in Central Ethiopia, 2017.

larger areas compared to the above studies. This low level of adherence can be averted by providing patients with meaningful information or health education about treatment adherence.

The findings of this research revealed that farmers were less likely to adhere to antihypertensive treatment compared to employed people. The Farmer is likely to be less educated and may have less information on the treatment adherence compared to the employed person $[44,45]$. Therefore, healthcare providers should prepare health education to their clients based on their level of awareness by a due emphasis on farmers.

In this study, respondents who had family support were more likely to adhere to their treatment compared to those who have no family support. This finding is similar to the studies conducted in Northern Ethiopia, Mekele [29], and Nigeria [46], and an absence of household support had a strong negative effect on adherence among hypertensive patients. Likewise, studies in Congo reported that patients who received no support from family members about taking their medications were more likely to be noncompliant than the others [47]. The reason for this could be family support is important in the long-term management of hypertension, which requires a life-long change in the lifestyle of the affected person. Strong perceived family support will improve their self-worth and motivation. It is plausible that a motivated hypertensive patient will adhere to therapeutic plans and, therefore, achieve better blood pressure control.

While there may be no clear symptoms of hypertension, understanding how patients perceive hypertension (their illness perceptions) is necessary because, without this, treatment may not be appropriately tailored to their needs and belief systems. Understanding individuals' perceptions 
TABLE 7: Effect of selected variable and other characteristics on adherence to antihypertensive treatment in Central Ethiopia, 2017.

\begin{tabular}{|c|c|c|c|c|c|c|}
\hline \multirow{2}{*}{ Variable } & \multicolumn{3}{|c|}{ Treatment adherence } & \multirow{2}{*}{ COR $(95 \%$ CI $)$} & \multirow{2}{*}{ AOR (95\% CI) } & \multirow{2}{*}{$P$ value } \\
\hline & Low & Medium & High & & & \\
\hline \multicolumn{7}{|l|}{ Sex } \\
\hline Male & 166 & 144 & 158 & 1 & 1 & \\
\hline Female & 153 & 170 & 198 & $1.26(0.99,1.58)$ & $1.21(0.94,1.55)$ & 0.133 \\
\hline \multicolumn{7}{|l|}{ Educational status } \\
\hline Illiterate & 98 & 113 & 131 & $1.57(1.10,2.24)^{*}$ & $1.39(0.94,2.06)$ & 0.103 \\
\hline Read and write & 52 & 42 & 148 & $1.31(0.86,1.99)$ & $1.30(0.83,2.03)$ & 0.245 \\
\hline Primary & 55 & 56 & 177 & $1.46(0.97,2.18)$ & $1.28(0.83,1.95)$ & 0.261 \\
\hline Secondary & 54 & 57 & 172 & $1.39(0.93,2.08)$ & $1.26(0.82,1.92)$ & 0.290 \\
\hline Diploma and above & 60 & 46 & 150 & 1 & 1 & \\
\hline \multicolumn{7}{|l|}{ Occupation } \\
\hline Gov. employed & 46 & 38 & 70 & 1 & 1 & \\
\hline Self-employed & 44 & 44 & 63 & $0.92(0.60,1.40)$ & $0.86(0.56,1.32)$ & 0.481 \\
\hline Farmer & 54 & 41 & 34 & $0.49(0.32,0.76)^{*}$ & $0.51(0.33,0.79)^{*}$ & 0.003 \\
\hline House wife & 108 & 108 & 109 & $0.69(0.49,0.99)^{*}$ & $0.71(0.49,1.02)$ & 0.068 \\
\hline Retired & 67 & 83 & 80 & $0.78(0.53,1.14)$ & $0.70(0.48,1.05)$ & 0.084 \\
\hline \multicolumn{7}{|l|}{ Frequency of visit } \\
\hline Once in a month & 159 & 180 & 212 & 1 & 1 & \\
\hline$>$ Once in month & 27 & 25 & 30 & $1.74(1.03,1.76)^{*}$ & $1.04(0.67,1.61)$ & 0.856 \\
\hline Once in 2 months & 42 & 17 & 33 & $1.17(0.75,1.86)$ & $0.73(0.47,1.14)$ & 0.164 \\
\hline Once in 3 months or more & 91 & 92 & 81 & $0.85(0.54,1.34)$ & $0.92(0.69,1.22)$ & 0.565 \\
\hline \multicolumn{7}{|l|}{ Family support } \\
\hline Yes & 235 & 252 & 295 & $1.52(1.14,2.02)^{*}$ & $1.65(1.23,2.22)^{*}$ & 0.001 \\
\hline No & 84 & 62 & 61 & 1 & 1 & \\
\hline \multicolumn{7}{|l|}{ Treatment control } \\
\hline Lower & 141 & 148 & 133 & 1 & 1 & \\
\hline Higher & 178 & 166 & 233 & $0.81(0.63,1.02)^{*}$ & $0.86(0.67,1.11)$ & 0.246 \\
\hline \multicolumn{7}{|l|}{ Perceived consequence } \\
\hline Lower & 162 & 156 & 142 & 1 & 1 & \\
\hline Higher & 157 & 158 & 214 & $1.18(1.17,1.88)^{*}$ & $1.51(1.17,1.95)^{* *}$ & 0.001 \\
\hline \multicolumn{7}{|l|}{ Cyclical perception } \\
\hline Lower & 162 & 173 & 209 & 1 & 1 & \\
\hline Higher & 157 & 141 & 147 & $0.77(0.61,0.98)^{*}$ & $0.76(0.58,0.95)^{*}$ & 0.019 \\
\hline \multicolumn{7}{|l|}{ Perceived susceptibility } \\
\hline Lower & 172 & 165 & 251 & 1 & 1 & \\
\hline Higher & 147 & 149 & 105 & $0.59(0.46,074)$ & $0.61(0.48,0.78)^{*}$ & 0.0001 \\
\hline \multicolumn{7}{|l|}{ Perceived severity } \\
\hline Lower & 138 & 105 & 96 & 1 & 1 & \\
\hline Higher & 181 & 209 & 260 & $1.73(1.36,2.21)^{*}$ & $1.42(1.09,1.86)^{*}$ & 0.010 \\
\hline \multicolumn{7}{|l|}{ Perceived benefit } \\
\hline Low & 76 & 78 & 72 & 1 & 1 & \\
\hline High & 243 & 236 & 284 & $1.17(0.89,1.54)$ & $1.09(0.82,1.46)$ & 0.547 \\
\hline \multicolumn{7}{|l|}{ Cues to action } \\
\hline Low & 171 & 184 & 208 & 1 & 1 & \\
\hline High & 148 & 130 & 148 & $0.86(0.69,1.09)$ & $0.89(0.69,1.115)$ & 0.373 \\
\hline
\end{tabular}

about their illnesses provide a key for developing effective strategies to cope with chronic illnesses. Perception of a lower ability to control a health threat can be a barrier to one's behavioral actions, or cognitive and emotional changes in illness representations because of somatic experiences. Higher perception of illness control is associated with lower anxiety, lower avoidance/denial of coping strategies, and positive reappraisal $[48,49]$. In the present study, among illness perception dimensions perceived consequences and cyclical perception had a significant association with treatment adherence.

The respondent's perception of the consequences of the disease was also positively related to treatment adherence, which was consistent with reports by other researchers $[50,51]$. The perception of consequences has been shown to improve motivation to adhere to antihypertensive treatment [52].

Patients' beliefs about how long their symptoms will last might impact their decisions to seek or not seek healthcare. Cyclical timeline refers to the belief of an individual that the expected symptoms will diminish after a certain period and then they might reappear at a certain time in the future. When the symptoms diminished, patients believe that it is cured and more likely to be nonadherent, including decreasing frequency and types of medication, taking medications intermittently, and deviating from the prescribed 
timing. In this regard, respondents who had higher cyclical perception were less likely to adhere to their treatment. The findings of this research were also supported by a study conducted by Chen et al. [49] indicated that a belief that the illness (hypertension) cyclical was associated with nonadherence to treatment.

In treating hypertension, understanding patient's beliefs about medication adherence is fundamental because hypertension is often silent and asymptomatic. Thus, patients might undermine its severity and the significance of its management $[27,30,53]$, leading to poor adherence to antihypertensive medication.

According to the Health Belief Model (HBM) people's perception of risk predicts their behaviors, low-risk perception leads to undesired behaviors [52]. Hypertensive patients with low-risk perception may have a risky lifestyle and poor adherence to prescribed medications, which significantly compromise the control of the condition $[54,55]$.

Under the constructs of the HBM, those with a higher perceived susceptibility showed better adherence to antihypertensive medications $[55,56]$. A study conducted among patients in South India found that medication adherence was significantly associated with all the six components of the HBM [57], and a Chinese study found that higher levels of perceived susceptibility, cues to action, and self-efficacy and a lower level of perceived barriers were significantly associated with better antihypertensive medication adherence [58]. However, in our study, only perceived susceptibility and perceived severity to hypertension showed a significant association with treatment adherence.

The importance of understanding treatment adherence to antihypertension medications in controlling the patient's blood pressure and reducing hypertension-related complications to clinical practice cannot be overemphasized. Healthcare providers have to be aware of and understand patient's beliefs about their illness and medications while caring for them and incorporating this belief in designing effective interventions to improve medication adherence through reducing barriers to taking medications.

Overall, $67.7 \%$ of patients were reporting a medium or high level of adherence to antihypertensive medication in this research. Among the study participants, farmers by occupation, having family support, perceived consequences, perceived cyclical nature of hypertension, perceived susceptibility, and perceived severity of the disease showed a significant association with treatment adherence. Illness perceptions and other beliefs may influence the actions of the individual and negatively or positively affect his or her health and taking medication. Therefore, it is important before and during treatment to assess patients' views about their illness, symptoms, and treatments.

\section{Data Availability}

The datasets generated and/or analyzed during the current study are not publicly available due to some privacy reasons, but part of the raw dataset will be available from the corresponding author upon reasonable request.

\section{Ethical Approval}

An ethical approval letter was obtained from the institutional review board of Arsi University. Each study participant was adequately informed about the purpose, method, anticipated benefit, and risk of the study by the data collectors. The respondents' right to refuse or withdraw from participating in the interview was fully maintained, and the information provided by each respondent is kept strictly confidential.

\section{Consent}

The respondents were informed about the purpose of the study, and their oral consent was obtained before the data collection. Anonymity was also maintained to ensure ensure confidentiality.

\section{Conflicts of Interest}

The authors declare that they have no conflicts of interest.

\section{Acknowledgments}

The authors would like to thank Arsi University for the support they made to realize this research, hospitals, data collectors, and all participants for their enthusiastic participation.

\section{References}

[1] A. Ahmed, M. Rahman, R. Hasan et al., "Hypertension and associated risk factors in some selected rural areas of Bangladesh," International Journal of Research in Medical Sciences, vol. 2, no. 3, pp. 925-931, 2014.

[2] S. M. Abebe, Y. Berhane, A. Worku, and A. Getachew, "Prevalence and associated factors of hypertension: a crossectional community based study in Northwest Ethiopia," PLoS One, vol. 10, no. 4, Article ID e0125210, 2015.

[3] K. T. Mills, J. D. Bundy, T. N. Kelly et al., "Global disparities of hypertension prevalence and control," Circulation, vol. 134, no. 6, pp. 441-450, 2016.

[4] WHO, "Global, regional, and national disability-adjusted lifeyears (DALYs) for 315 diseases and injuries and healthy life expectancy (HALE), 1990-2015: a systematic analysis for the global burden of disease study 2015," The Lancet, vol. 388, no. 10053, pp. 1603-1658, 2016.

[5] World Health Organization, Raised Blood Pressure Situation and Trends, World Health Organization, Geneva, Switzerland, 2014.

[6] P. A. James, S. Oparil, B. L. Carter et al., "2014 evidence-based guideline for the management of high blood pressure in adults," JAMA, vol. 311, no. 5, pp. 507-520, 2014.

[7] L. C. van Vark, M. Bertrand, K. M. Akkerhuis et al., "Angiotensin-converting enzyme inhibitors reduce mortality in hypertension: a meta-analysis of randomized clinical trials of renin-angiotensin-aldosterone system inhibitors involving 158998 patients," European Heart Journal, vol. 33, no. 16, pp. 2088-2097, 2012.

[8] S. M. Cohen, "Concept analysis of adherence in the context of cardiovascular risk reduction," Nursing Forum, vol. 44, no. 1, pp. 25-36, 2009. 
[9] V. Fung, J. Huang, R. Brand, J. P. Newhouse, and J. Hsu, "Hypertension treatment in a medicare population: adherence and systolic blood pressure control," Clinical Therapeutics, vol. 29, no. 5, pp. 972-984, 2007.

[10] L. D. Esposti, S. Saragoni, S. Benemei et al., "Adherence to antihypertensive medications and health outcomes among newly treated hypertensive patients," ClinicoEconomics and Outcomes Research, vol. 3, pp. 47-54, 2011.

[11] K. Matsumura, H. Arima, M. Tominaga et al., "Impact of antihypertensive medication adherence on blood pressure control in hypertension: the COMFORT study," QJM, vol. 106, no. 10, pp. 909-914, 2013.

[12] S. Shin, H. Song, S.-K. Oh, K. E. Choi, H. Kim, and S. Jang, "Effect of antihypertensive medication adherence on hospitalization for cardiovascular disease and mortality in hypertensive patients," Hypertension Research, vol. 36, no. 11, pp. 1000-1005, 2013.

[13] P.-H. Wu, C.-Y. Yang, Z.-L. Yao, W.-Z. Lin, L.-W. Wu, and C.-C. Chang, "Relationship of blood pressure control and hospitalization risk to medication adherence among patients with hypertension in Taiwan," American Journal of Hypertension, vol. 23, no. 2, pp. 155-160, 2010.

[14] M. Krousel-Wood, S. Thomas, P. Muntner, and D. E. Morisky, "Medication adherence: a key factor in achieving blood pressure control and good clinical outcomes in hypertensive patients," Current Opinion in Cardiology, vol. 19, no. 4, pp. 357-362, 2004.

[15] M. A. Krousel-Wood, P. Muntner, T. Islam, D. E. Morisky, and L. S. Webber, "Barriers to and determinants of medication adherence in hypertension management: perspective of the cohort study of medication adherence among older adults," Medical Clinics of North America, vol. 93, no. 3, pp. 753-769, 2009.

[16] G. Mazzaglia, E. Ambrosioni, M. Alacqua et al., "Adherence to antihypertensive medications and cardiovascular morbidity among newly diagnosed hypertensive patients," Circulation, vol. 120, no. 16, pp. 1598-1605, 2009.

[17] M. C. Sokol, K. A. Verbrugge, and R. S. Epstein, "Impact of medication adherence on hospitalization risk and healthcare cost," Medical Care, vol. 43, no. 6, pp. 521-530, 2005.

[18] W. F. Gellad, J. L. Grenard, and Z. A. Marcum, "A systematic review of barriers to medication adherence in the elderly: looking beyond cost and regimen complexity," The American Journal of Geriatric Pharmacotherapy, vol. 9, no. 1, pp. 11-23, 2011.

[19] C. A. Jackson, J. Clatworthy, A. Robinson, and R. Horne, "Factors associated with non-adherence to oral medication for inflammatory bowel disease: a systematic review," American Journal of Gastroenterology, vol. 105, no. 3, pp. 525-539, 2010.

[20] E. Broadbent, L. Donkin, and J. C. Stroh, "Illness and treatment perceptions are associated with adherence to medications, diet, and exercise in diabetic patients," Diabetes Care, vol. 34, no. 2, pp. 338-340, 2011.

[21] R. Horne, S. C. E. Chapman, R. Parham, N. Freemantle, A. Forbes, and V. Cooper, "Understanding patients' adherence-related beliefs about medicines prescribed for long-term conditions: a meta-analytic review of the necessity-concerns framework," PLoS One, vol. 8, no. 12, Article ID e80633, 2013.

[22] A. S. Gadkari and C. McHorney, "Unintentional non-adherence to chronic prescription medications: how unintentional is it really?" BMC Health Services Research, vol. 12, no. 98 , pp. 1-12, 2012.

[23] B. Schüz, C. Marx, S. Wurm et al., "Medication beliefs predict medication adherence in older adults with multiple illnesses,"
Journal of Psychosomatic Research, vol. 70, no. 2, pp. 179-187, 2011.

[24] I. J. Marshall, C. D. A. Wolfe, and C. McKevitt, "Lay perspectives on hypertension and drug adherence: systematic review of qualitative research," BMJ, vol. 345, p. e3953, 2012.

[25] World Health Organization, A Global Brief on Hypertension, Silent Killer, Global Public Health Crisis, World Health Organization, Geneva, Switzerland, 2013.

[26] M. R. Akpa, D. I. Agomuoh, and O. J. Odia, "Drug compliance among hypertensive patients in Port Harcourt, Nigeria," Nigerian Journal of Medicine, vol. 14, no. 1, pp. 55-57, 2005.

[27] K. Petrie and J. Weinman, "Why illness perceptions matter," Clinical Medicine, vol. 6, no. 6, pp. 536-539, 2006.

[28] A. D. Ambaw, G. A. Alemie, S. M. Yohannes, and Z. B. Mengesha, "Adherence to antihypertensive treatment and associated factors among patients on follow up at university of Gondar hospital, Northwest Ethiopia," BMC Public Health, vol. 12, no. 282, pp. 1-6, 2012.

[29] M. A. Ali, M. L. Bekele, and G. Teklay, "Antihypertensive medication non-adherence and its determinants among patients on follow up in public hospitals in Northern Ethiopia," International Journal of Clinical Trials, vol. 1, no. 3, pp. 95104, 2014.

[30] F. E. S. Girma and F. Alemseged, "A compliance with antihypertensive treatment and associated factors among hypertensive patients on follow-up in Jimma university specialized hospital, Jimma, south west Ethiopia: a quantitative cross sectional study," Journal of Hypertension, vol. 3, no. 5, pp. 1-6, 2014.

[31] H. A. A. M. Hareri and T. Asefaw, "Assessments of adherence to hypertension managements and its influencing factors among hypertensive patients attending Tikur Anbessa hospital chronic follow up unit, Addis Ababa, Ethiopia-a crosssectional study," International Journal of Pharmaceutical Sciences and Research, vol. 4, no. 3, pp. 1086-1095, 2013.

[32] A. Tibebu, D. Mengistu, and L. M. Bulto, "Adherence to prescribed antihypertensive medications and associated factors for hypertensive patients attending chronic follow-up units of selected public hospitals in Addis Ababa," International Journal of Health Sciences, vol. 11, no. 4, pp. 47-52, 2017.

[33] R. Moss-Morris, J. Weinman, K. Petrie, R. Horne, L. Cameron, and D. Buick, "The revised illness perception questionnaire (IPQ-R)," Psychology \& Health, vol. 17, no. 1, pp. 1-16, 2002.

[34] D. E. Morisky, A. Ang, M. Krousel-Wood, and H. J. Ward, "Predictive validity of a medication adherence measure in an outpatient setting," The Journal of Clinical Hypertension, vol. 10, no. 5, pp. 348-354, 2008.

[35] N. S. Ali, "Prediction of coronary heart disease preventive behaviors in women: a test of the Health Belief Model," Women \& Health, vol. 35, no. 1, pp. 83-95, 2002.

[36] D. R. Berlowitz, C. G. Foy, L. E. Kazis et al., "Effect of intensive blood-pressure treatment on patient-reported outcomes," New England Journal of Medicine, vol. 377, no. 8, pp. 733-744, 2017.

[37] A. P. Bress, B. K. Bellows, J. B. King et al., "Cost-effectiveness of intensive versus standard blood-pressure control," New England Journal of Medicine, vol. 377, no. 8, pp. 745-755, 2017.

[38] A. Bowling, "Mode of questionnaire administration can have serious effects on data quality," Journal of Public Health, vol. 27, no. 3, pp. 281-291, 2005. 
[39] A. A. Akintunde and T. S. Akintunde, "Antihypertensive medications adherence among Nigerian hypertensive subjects in a specialist clinic compared to a general outpatient clinic," Annals of Medical and Health Sciences Research, vol. 5, no. 3, pp. 173-178, 2015.

[40] E. A. Ibrahim, "Level of adherence to lifestyle changes and medications among male hypertensive patients in two hospitals in Taif; Kingdom of Saudi Arabia," International Journal of Pharmacy and Pharmaceutical Sciences, vol. 7, no. 4, pp. 168-172, 2015.

[41] G. Okwuonu Chimezie, V. Uwanurochi Ndidiamaka, J. B. Chimezie Oluchi, S. Ogah Okechukwu, U. Mbanaso Augustus, and O. Odigwe Clement, "Adherence to antihypertensive medication and its correlates among individuals with hypertension in a semi-urban community of Southern Nigeria," Journal of Medicine and Biomedical Research, vol. 14, no. 1, pp. 5-17, 2015.

[42] P. Misra, H. R. Salve, R. Srivastava, S. Kant, and A. Krishnan, "Adherence to treatment among hypertensive individuals in a rural population of North India," Indian Journal of Community Health, vol. 29, no. 2, pp. 176-181, 2017.

[43] J. Venkatachalam, S. Abrahm, Z. Singh, P. Stalin, and G. Sathya, "Determinants of patient's adherence to hypertension medications in a rural population of Kancheepuram district in Tamil Nadu, South India," Indian Journal of Community Medicine, vol. 40, no. 1, pp. 33-37, 2015.

[44] J. S. Gonzalez, A. W. Batchelder, C. Psaros, and S. A. Safren, "Depression and HIV/AIDS treatment nonadherence: a review and meta-analysis," JAIDS Journal of Acquired Immune Deficiency Syndromes, vol. 58, no. 2, pp. 181-187, 2011.

[45] M. Sousa-Ribeiro, M. Sverke, and J. L. Coimbra, "Perceived quality of the psychosocial environment and well-being in employed and unemployed older adults: the importance of latent benefits and environmental vitamins," Economic and Industrial Democracy, vol. 35, no. 4, pp. 629-652, 2014.

[46] P. E. O. Social, "Support and management of hypertension in south-west Nigeria," Cardiovascular Journal of Africa, vol. 26, no. 1, pp. 29-33, 2015.

[47] B. Nsitou, B. Drame, M. S. Ikama, and B. B. Kaboru, "Patientsrelated predictors of poor adherence to antihypertensive treatment in Congo-brazzaville: a cross- sectional study," Global Journal of Medicine \& Public Health, vol. 2, 2013.

[48] D. C. Jessop and D. R. Rutter, "Adherence to asthma medication: the role of illness representations," Psychology \& Health, vol. 18, no. 5, pp. 595-612, 2003.

[49] S.-L. Chen, J.-C. Tsai, and W.-L. Lee, "The impact of illness perception on adherence to therapeutic regimens of patients with hypertension in Taiwan," Journal of Clinical Nursing, vol. 18, no. 15, pp. 2234-2244, 2009.

[50] W.-W. Li, C.-T. Kuo, S.-L. Hwang, and H.-T. Hsu, "Factors related to medication non-adherence for patients with hypertension in Taiwan," Journal of Clinical Nursing, vol. 21, no. 13-14, pp. 1816-1824, 2012.

[51] S. Maharjan, T. Chinnawong, and C. Kritpracha, "Illness perception among patients with hypertension in Nepal," GSTF Journal of Nursing and Health Care, vol. 4, no. 2, pp. 79-85, 2017.

[52] B. Raingruber, Contemporary Health Promotion in Nursing, Jones \& Bartlett Learning, Burlington, MA, USA, 2013.

[53] N. R. Campbell, R. Petrella, and J. Kaczorowski, "Public education on hypertension: a new initiative to improve the prevention, treatment and control of hypertension in Canada," Canadian Journal of Cardiology, vol. 22, no. 7, pp. 599-603, 2006.
[54] J. Mpinda, J. Tumbo, I. Govender, and B. Mills, "The knowledge and beliefs of hypertensive patients attending Katleho district hospital in free state province, South Africa, about their illness," South African Family Practice, vol. 56, no. 4, pp. 229-234, 2014.

[55] R. Oruganti, S. Paidipati, and M. Dinaker, "Knowledge about hypertension: a comparison between hypertensive patients and their normotensive counterparts," IOSR Journal of Nursing and Health Sciences, vol. 7, no. 6, pp. 27-36, 2018.

[56] A. Kamran, S. S. Ahari, M. Biria, A. Malepour, and H. Heydari, 'Determinants of patient' adherence to hypertension medications: application of health belief model among rural patients," Annals of Medical and Health Sciences Research, vol. 4, no. 6, pp. 922-927, 2014.

[57] A. U. Shameena, S. Badiger, and S. Nanjesh Kumar, "Medication adherence and health belief model among hypertensive patients attending rural health centers of a tertiary care hospital in South India," International Journal of Community Medicine and Public Health, vol. 4, no. 4, pp. 1159-1165, 2017.

[58] Z. Yue, C. Li, Q. Weilin, and W. Bin, "Application of the health belief model to improve the understanding of antihypertensive medication adherence among Chinese patients," Patient Education and Counseling, vol. 98, no. 5, pp. 669-673, 2015. 